\title{
Detection and Classification of Ventricular Tachycardia Using SVM
}

\author{
Palimaru Aparna ${ }^{1}$, Praveen Mirajkar ${ }^{2}$, Raghavendra Prabhu ${ }^{3}$ \\ PG Scholar, Electrical, NMAMIT, Nitte, India ${ }^{1}$ \\ R\&D Engineer, Caliber Assurance Services, Bangalore, India ${ }^{2}$ \\ Assistant Professor, Electrical, NMAMIT, Nitte, India ${ }^{3}$
}

\begin{abstract}
Ventricular Tachycardia is an abnormal heart rhythm that initiates in the ventricles. Non-sustained VTach lasts for few seconds and Sustained VTach lasts for few minutes or even hours. Sustained VTach is dangerous compared to Non-sustained VTach and if it is not treated, it often progresses to Ventricular Fibrillation. VTach is serious in people suffering with heart disease and is associated with more symptoms than other types of arrhythmia. Accurate prediction of Ventricular Tachycardia can be obtained by observing the changes in $T$ wave, ST segment and QT interval which are the indicators for cardiac instability. In this paper, we present an algorithm that detects Ventricular Tachycardia by using morphological features of electrical signal of ventricles activity obtained from ECG. Classification of features is carried out by using Support Vector Machines (SVM). The proposed algorithm is tested on 22 records including Normal Sinus Rhythm and Ventricular Tachycardia which are collected from MIT-BIH Normal Sinus Rhythm database and CU Ventricular Tachyarrhythmia database respectively and satisfactory result is obtained as the $92.31 \%$ Specificity, $100 \%$ Sensitivity and $95.45 \%$ Accuracy is obtained.
\end{abstract}

Keywords: Normal Sinus Rhythm, Ventricular Tachycardia, Adaptive Threshold, Hilbert Transform, SVM.

\section{INTRODUCTION}

Cardiovascular disease is one of the most common causes of death. Cardiac arrhythmia or dysrhythmia represents a group of conditions in which the electrical activity of the heart is irregular, too fast or too slow than normal. Heart rate that is too fast $(\mathrm{HR}>100 \mathrm{bpm})$ is called Tachycardia and the heart rate that is too slow $(\mathrm{HR}<60 \mathrm{bpm})$ is called Bradycardia. The heart may not be able to pump enough blood to the body during an arrhythmia. Lack of blood flow can damage the parts of body like brain, heart and other organs. All arrhythmias are not life-threatening but Ventricular arrhythmias can cause cardiac arrest. Arrhythmias that occur in the upper chambers of the heart are called Atrial Arrhythmias, that occur in AV node of the heart are called Atrio-Ventricular Arrhythmias and that occur in lower chambers of the heart are called Ventricular Arrhythmias.Ventricular Tachycardia is the most serious cardiac arrhythmia among Ventricular arrhythmias which if not treated properly can lead to death. Ventricular Tachycardia is an abnormal heart rhythm that originates from the fast improper electrical activity of the ventricles. Electrocardiography is a non-invasive test for recording the electrical activity of the heart. It is a method to diagnose heart diseases using ECG signals. ECG signal comprises of $\mathrm{P}$ wave, QRS complex and $\mathrm{T}$ wave. In the normal ECG signal, the main parameters which are examined include the shape, the duration, and the correlation between the $\mathrm{P}$ wave, QRS complex, $\mathrm{T}$ wave components and R-R Interval. The variations in these parameters are used to identify the type of illness of the heart. ECG waveform for a single cardiac cycle is shown in Fig. 1.

This paper is organized as follows: Proposed Algorithm is described in Section II. The results are showed in Section III and conclusion is presented in Section IV.

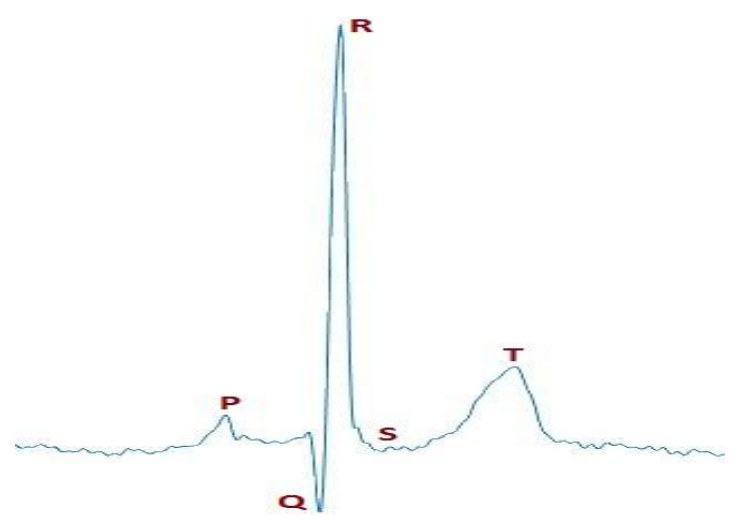

Fig.1ECG waveform for a single cardiac cycle

\section{PROPOSED ALGORITHM}

The proposed algorithm consists of four steps: Preprocessing of ECG signal, QRS complex and T wave 


\title{
International Journal of Innovative Research in Electrical, Electronics, Instrumentation and Control Engineering
} NCAEE 2017

\author{
National Conference on Advances in Electrical Engineering \\ NMAM Institute of Technology, Nitte \\ Vol. 5, Special Issue 2, April 2017
}

detection, extraction of features and classifying it Where the vector $h$ is filled as shown in Eq. (2). The usingSupport Vector Machines (SVM). Block diagram of vector f stores the Fast Fourier Transform (FFT) of the proposed algorithm is shown in Fig. 2.

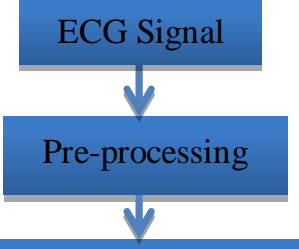

QRS complex and T wave Detection

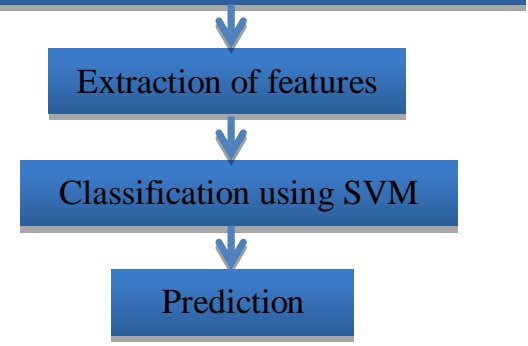

Fig. 2 Block diagram of Proposed Algorithm

\section{A. Pre-processing of ECG signal \\ 1) ECG filtering:}

The main aim of pre-processing stage is to remove noise from the ECG signal for accurate analysis. Raw ECG signal contains Baseline Wandering noise caused by respiration, Power-line Interference $(50 \mathrm{~Hz})$, high frequency random noises caused my muscular activity. To remove muscular noise we use band pass filter which will also maximize QRS complex. The band lies between $5 \mathrm{~Hz}-$ $25 \mathrm{~Hz}$. This will remove high frequency and baseline wandering noises. It also suppresses the $\mathrm{P}$ wave and $\mathrm{T}$ wave.

\section{2) Differentiation:}

The first derivative is applied to indicate the minimum and high slope points of the ECG signal i.e., the falling of signal from $\mathrm{R}$ point to $\mathrm{S}$ point and the rising of signal from $\mathrm{Q}$ point to $\mathrm{R}$ point respectively. The first derivative 2point central difference is calculated by using the below equation

$$
\mathrm{y}(\mathrm{k})=\frac{1}{2 \Delta \mathrm{t}}(\mathrm{y}(\mathrm{k}+1)-\mathrm{y}(\mathrm{k}-1)), \quad \mathrm{k}=0,1,2, \ldots, \mathrm{N}-1
$$

Where $2 \Delta \mathrm{t}$ is the sampling frequency and $\mathrm{N}$ is the total number of samples. The derivative output of the filtered signal removes motion artifacts and baseline drifts.

\section{B. QRS complex and T wave Detection \\ 1) Hilbert Transform:}

Hilbert Transform method is applied to the filtered ECG signal. The Hilbert Transform for the discrete time series $y(k)$ is defined as in Eq. (1)

$$
H(k)=y_{H}(k)=F F T^{-1}(f(k) * h(i))
$$

$y(k)$ signal and $\mathrm{FFT}^{-1}$ is the Inverse Fast Fourier Transform.

$$
\begin{gathered}
0 \text { for } i=(N / 2)+2, \ldots, N \\
2 \text { for } i=2,3, \ldots,(N / 2) \\
1 \text { for } i=1,(N / 2)+1
\end{gathered}
$$

The analytic signal $z(k)$ is given in Eq. (3). It is the preenvelope of the original signal $y(k)$.

$$
z(k)=y(k)+j y_{H}(k)
$$

The envelope $a(k)$ of $z(k)$ is described in Eq. (4). It is also considered as instantaneous magnitude of $z(k)$.

$$
a(k)=\sqrt{y^{2}(k)+y_{H}^{2}(k)}
$$

The instantaneous phase angle in the complex plane is defined as in Eq. (5).

$$
\theta(k)=\arctan \left(\frac{y_{H}(k)}{y(k)}\right)
$$

\section{2) Adaptive Threshold Technique:}

Adaptive Threshold Technique is used to detect QRS complex and T wave. Adaptive Threshold is a method to detect $R$ peaks of the ECG signal. This method is performed by using a pair of threshold limits called upper threshold limit and lower threshold limit.

The upper and lower thresholds are defined by Eq. (6) and Eq. (7) respectively, where $\alpha$ is the maximum value attained $y(k)$ on the point $\mathrm{k}=1, \ldots, \mathrm{N}$

$$
\begin{aligned}
u_{t h} & =0.6 \times \alpha \\
l_{t h} & =0.4 \times \alpha
\end{aligned}
$$

The threshold values are updated in iteration time. The number of peaks detected above the lower and upper threshold limits are calculated. This process continues until the number of peaks detected above lower threshold limit equals the number of peaks detected above upper threshold limit.

$u_{t h}$ and $l_{t h}$ are updated using Eq. (8) and Eq. (9)

$$
\begin{aligned}
& u_{t h}(k+1)=u_{t h}(k)-w \Delta \\
& l_{t h}(k+1)=l_{t h}(k)+w \Delta
\end{aligned}
$$

Where the error weight $\mathrm{w}=0.125$ and $\Delta=u_{t h}-l_{t h}$ is the difference between defined two limits.

$\mathrm{T}$ wave is detected by considering a window with beginning of $70 \mathrm{~ms}$ after $\mathrm{R}$ peak and ends in $350 \mathrm{~ms}$ after $\mathrm{R}$ peak and find the maximum point. $\mathrm{A}$ window of appreciable length has been considered before and after the $\mathrm{T}$ peak location and find their minimum value for the detection of beginning and ending of $\mathrm{T}$ wave. $\mathrm{R}$ point detection in NSR and VTach are shown in Fig. 3 and Fig. 4 respectively. QRS complex and $\mathrm{T}$ wave detection waveform is shown in Fig. 5. 
National Conference on Advances in Electrical Engineering

NMAM Institute of Technology, Nitte

Vol. 5, Special Issue 2, April 2017

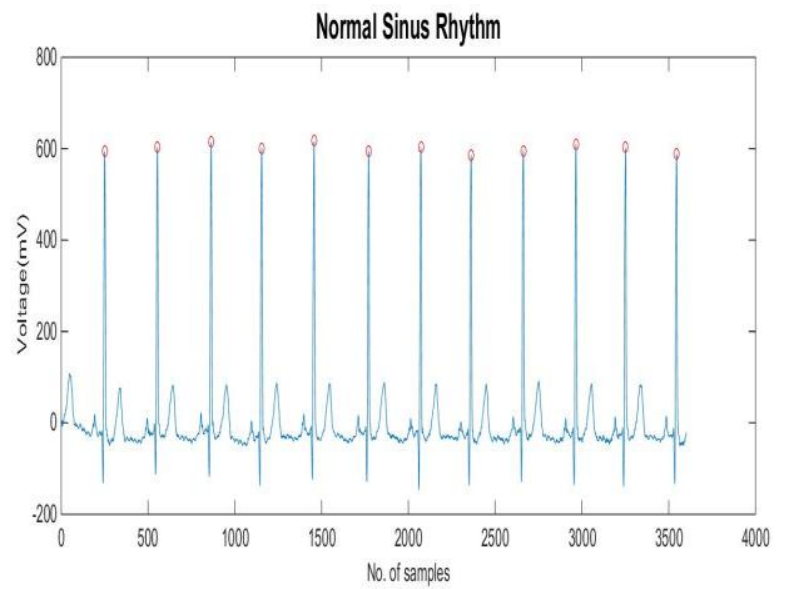

Fig. $3 \mathrm{R}$ point detection in Normal Sinus Rhythm Signal

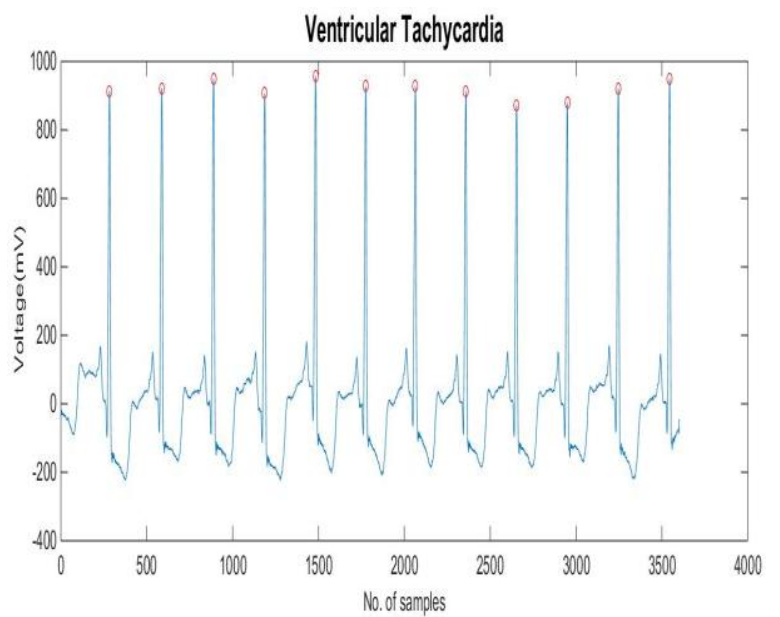

Fig. 4 R point detection in Ventricular Tachycardia Signa

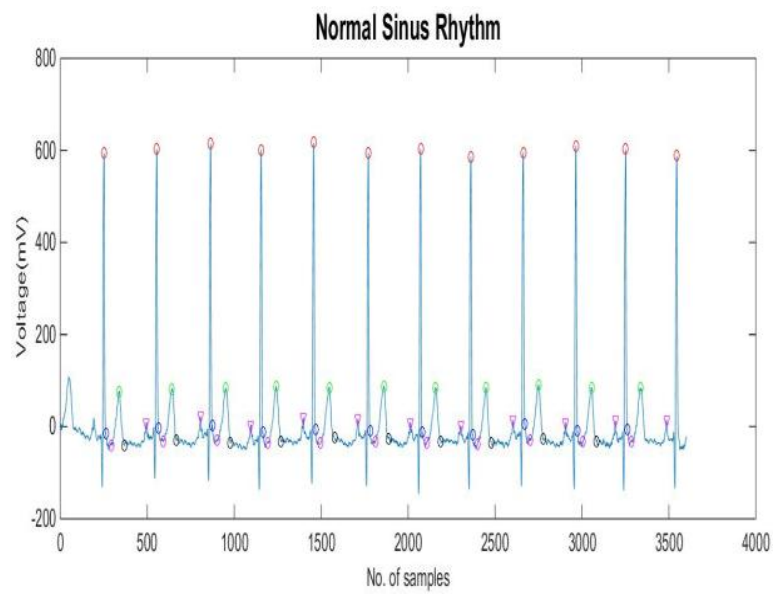

Fig. 5 QRS Complex and T wave detection

C. Extraction of features

We extract some morphological features for the detection of Ventricular Tachycardia. We selected nine features to distinguish between normal and abnormal. These features are as follows:
1) Total $\mathrm{T}$ wave area:Calculate the area under $\mathrm{T}$ wave by using the formula,

$$
\text { Tarea }_{k}=\sum_{n=\text { TStart }_{k}}^{\text {Tend }_{k}} T_{k}(n)
$$

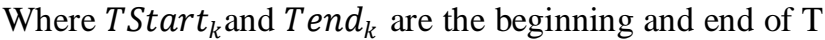
wave in each beat, $\mathrm{k}$ is the beat number and $T_{k}(n)$ is $\mathrm{T}$ wave in the $\mathrm{k}^{\text {th }}$ beat.

2) Ascending $T$ wave area:Calculate the area under the ascending portion of $\mathrm{T}$ wave.

$$
\text { Tareaup }_{\mathrm{k}}=\sum_{\mathrm{n}=\text { Tstart }_{\mathrm{k}}}^{\text {Tpeak }_{\mathrm{k}}} \mathrm{T}_{\mathrm{k}}(\mathrm{n})
$$

Where Tstart $\mathrm{k}_{\mathrm{k}}$ and Tpeak $_{\mathrm{k}}$ are the beginning and peak of $\mathrm{T}$ wave in each beat respectively, $T_{k}(n)$ is $T$ wave in the $\mathrm{k}^{\text {th }}$ beat, $\mathrm{k}$ is the beat number.

3) Descending $T$ wave area: Calculate the area under the descending portion of $\mathrm{T}$ wave.

$$
\text { Tareadown }_{\mathrm{k}}=\sum_{\mathrm{n}=\text { Tpeak }_{\mathrm{k}}}^{\text {Tend }_{\mathrm{k}}} \mathrm{T}_{\mathrm{k}}(\mathrm{n})
$$

Where Tpeak $\mathrm{k}_{\mathrm{k}}$ and Tend $\mathrm{k}_{\mathrm{k}}$ are the peak and end of $\mathrm{T}$ wave in each beat respectively, $T_{k}(n)$ is $T$ wave in the $k^{\text {th }}$ beat, $k$ is the beat number.

4) TQ Interval: Calculate the interval between end of $\mathrm{T}$ wave in each beat and $\mathrm{Q}$ wave in next beat.

$$
\mathrm{TQ}_{\mathrm{k}}=\mathrm{Q}_{\mathrm{k}+1}-\mathrm{T}_{\mathrm{k}}
$$

5) QT Interval: Calculate the interval between Q wave in each beat and end of $\mathrm{T}$ wave in same beat.

$$
\mathrm{QT}_{\mathrm{k}}=\mathrm{T}_{\mathrm{k}}-\mathrm{Q}_{\mathrm{k}}
$$

6) ST segment slope: This is calculated by using the formula given below:

$$
\operatorname{Abs}\left(\operatorname{STslope}_{\mathrm{k}}\right)=\frac{\mathrm{y}\left(\operatorname{Jend}_{\mathrm{k}}\right)-\mathrm{y}\left(\mathrm{J}_{\mathrm{k}}\right)}{\left(\operatorname{Jend}_{\mathrm{k}}-\mathrm{J}_{\mathrm{k}}\right)}
$$

Where $\mathrm{Abs}\left(\mathrm{STslope}_{\mathrm{k}}\right)$ is absolute value of ST segment slope in $\mathrm{k}^{\text {th }}$ beat, $\mathrm{y}\left(\mathrm{Jend}_{\mathrm{k}}\right)$ and $\mathrm{y}\left(\mathrm{J}_{\mathrm{k}}\right)$ are the amplitude of last point of ST segment and $\mathrm{J}$ point respectively, Jend $_{\mathrm{k}}$ and $\mathrm{J}_{\mathrm{k}}$ are the location of last point of ST segment and $\mathbf{J}$ point respectively.

7) $T$ wave alternans: Calculate the difference between the $\mathrm{T}$ wave peak amplitude of each beat and its previous beat.

$$
\operatorname{Abs}\left(\mathrm{TWA}_{\mathrm{k}}\right)=\mathrm{Tamp}_{\mathrm{k}}-\mathrm{Tamp}_{\mathrm{k}-1}
$$


National Conference on Advances in Electrical Engineering

NMAM Institute of Technology, Nitte

Vol. 5, Special Issue 2, April 2017

Where Abs $\left(\mathrm{TWA}_{\mathrm{k}}\right)$ is absolute value of $\mathrm{T}$ wave alternans, Tamp $_{\mathrm{k}}$ and $\mathrm{Tamp}_{\mathrm{k}-1}$ are the values of $\mathrm{T}$ wave peak in each beat and its previous beat respectively.

8) Descending slope of $\mathrm{T}$ wave: Calculate the value of line connecting $\mathrm{T}$ wave peak in each beat to end of $\mathrm{T}$ wave.

$$
\text { AbsTslopedown } n_{\mathrm{k}}=\frac{\text { Tamp }_{\mathrm{k}}-\text { Tend }_{\mathrm{k}}}{\text { Tloc }_{\mathrm{k}}-\text { Tendloc }_{\mathrm{k}}}
$$

Where AbsTslopedown $n_{k}$ is absolute value of descending slope of $\mathrm{T}$ wave, Tamp $\mathrm{k}_{\mathrm{k}}$ and Tend $\mathrm{k}_{\mathrm{k}}$ are the peak and end values of $\mathrm{T}$ wave in each beat respectively, $\mathrm{Tloc}_{\mathrm{k}}$ and Tendloc $\mathrm{k}_{\mathrm{k}}$ are the location of $\mathrm{T}$ peak and end of $\mathrm{T}$ wave respectively.

9) Beat- beat correlation of $\mathrm{T}$ wave:This evaluates the resemblance degree of equal and symmetric samples of $\mathrm{T}$ wave in consecutive beats by calculating the cross correlation coefficient as follow:

$$
\mathrm{CR}_{\mathrm{T}_{\mathrm{k}}}=\frac{\sum_{\mathrm{n}=1}^{\mathrm{N}} \mathrm{T}_{\mathrm{k}}(\mathrm{n}) * \mathrm{~T}_{\mathrm{k}-1}(\mathrm{n})}{\sqrt{\sum_{\mathrm{n}=1}^{\mathrm{N}} \mathrm{T}_{\mathrm{k}}(\mathrm{n})^{2}} \sqrt{\sum_{\mathrm{n}=1}^{\mathrm{N}} \mathrm{T}_{\mathrm{k}-1}(\mathrm{n})^{2}}}
$$

Where $\mathrm{CR}_{\mathrm{T}_{\mathrm{k}}}$ is cross correlation coefficient of two consecutive $\mathrm{T}$ wave, $\mathrm{k}$ is beat number, $\mathrm{N}$ is number of $\mathrm{T}$ waves, $T_{k}(n)$ and $T_{k-1}(n)$ are the $T$ wave in $k^{\text {th }}$ beat and $(\mathrm{k}-1)^{\text {th }}$ beat respectively.

\section{Classification of features using SVM}

The presence of Arrhythmia is detected by Support Vector Machine classifier. SVM maximizes the geometrical margin and minimizes the empirical classification error. In this method we used nine features to classify VTach from Normal Sinus Rhythm(NSR) by using two-class SVM. Feature vectors along with the known class are provided to SVM, called the training data. This training data is used to develop mathematical function called hyper-plane for the separation of either class. Then the hyper-plane is applied to the new feature vector of unknown classes to distinguish between VTach and NSR.

\section{III.RESULT}

Proposed method is tested with Normal Sinus Rhythm and Ventricular Tachycardia beats taken from MIT-BIH Normal Sinus Rhythm and CU Ventricular Tachyarrhythmia database respectively. It was implemented on 16 records of NSR and 18 records of VTach, all of them with 10secs length and resampled to $360 \mathrm{~Hz}$. Out of 34 records, 12 of them are chosen as training data and 22 of them are chosen as test data. All the features are classified using Support Vector Machines with Radial Basis Function Kernel in MATLAB and Sensitivity, Specificity, Accuracy are calculated to evaluate the performance of the proposed method.

$$
\begin{aligned}
& \text { These three parameters } \\
& \text { Sensitivity }=\frac{\mathrm{TP}}{\mathrm{TP}+\mathrm{FN}}
\end{aligned}
$$

Specificity $=\frac{\mathrm{TN}}{\mathrm{TN}+\mathrm{FP}}$

Accuracy $=\frac{\mathrm{TP}+\mathrm{TN}}{\mathrm{TP}+\mathrm{TN}+\mathrm{FP}+\mathrm{FN}}$

Where TP is true positive detection, $\mathrm{TN}$ is true negative detection, FP is false positive detection, and $\mathrm{FN}$ is false negative detection. Sensitivity, Specificity, Accuracy ofthis method with SVM classification is $100 \%, 92.31 \%$, $95.45 \%$ respectively.

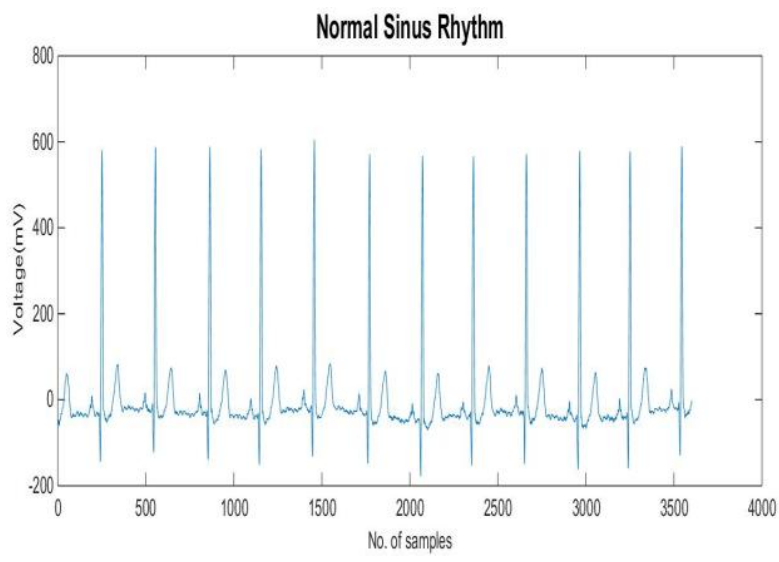

Fig. 6Normal Sinus Rhythm Signal

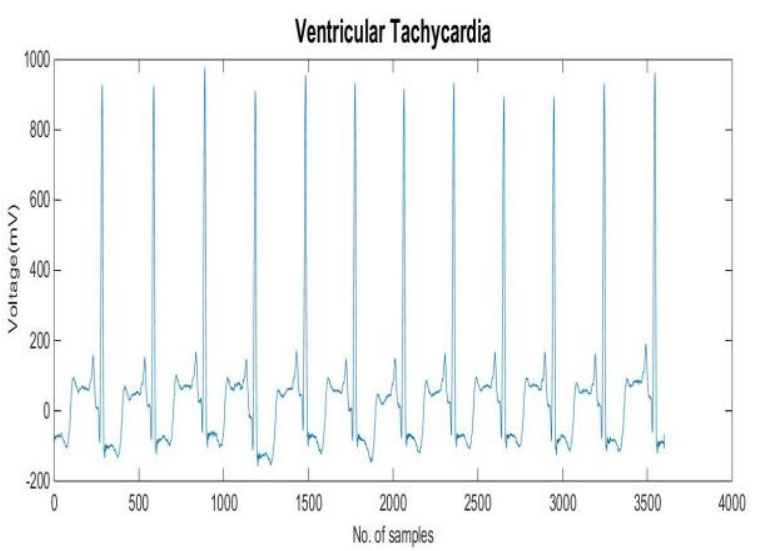

Fig. 7Ventricular Tachycardia Signal

TABLE I Database

\begin{tabular}{|c|c|c|}
\hline Signal & Database & Records \\
\hline $\begin{array}{c}\text { Normal Sinus } \\
\text { Rhythm }\end{array}$ & $\begin{array}{c}\text { MIT-BIH } \\
\text { Normal Sinus } \\
\text { Rhythm }\end{array}$ & 16 \\
\hline Ventricular \\
Tachycardia & $\begin{array}{c}\text { CU Ventricular } \\
\text { Tachyarrhythmia }\end{array}$ & 18 \\
\hline
\end{tabular}


National Conference on Advances in Electrical Engineering

NMAM Institute of Technology, Nitte

Vol. 5, Special Issue 2, April 2017

TABLE III Classification Results

\begin{tabular}{|c|c|c|c|}
\hline Signal & Training & Testing & $\begin{array}{c}\text { Correctly } \\
\text { classified }\end{array}$ \\
\hline $\begin{array}{c}\text { Normal Sinus } \\
\text { Rhythm }\end{array}$ & 6 & 10 & 9 \\
\hline $\begin{array}{c}\text { Ventricular } \\
\text { Tachycardia }\end{array}$ & 6 & 12 & 12 \\
\hline
\end{tabular}

\section{IV.CONCLUSION}

Proposed method is developed to distinguish between Ventricular Tachycardia and Normal Sinus Rhythm from their ECG waveforms. We have extracted nine features using ventricular activity part of ECG signal. These features are classified using SVM and accurate results are obtained for predicting the onset of VTach event. High performance of the results demonstrates that the proposed method can be used as reliable tool to predict Ventricular Tachycardia.

\section{ACKNOWLEDGMENT}

I would like to use this opportunity to thank and express my deepest gratitude to Jayavanth Kamath, CEO of Caliber Assurance Services who allowed me to carry out project at their esteemed organization. I would like to thank Mr. Raghavendra Prabhu, Assistant Professor of Department E\&E for his useful guidance and support. I would like to express my deepest gratitude and special thanks to Mr. Praveen Mirajkar, R\&D Engineer, Caliber Assurance Services for taking part in useful decision and giving necessary advices and guidance. I would like to express my sincere gratitude towards our Head of the Department of E\&E Dr. NageshPrabhu and our PG coordinator Prof. K. VasudevaShettighar for their support.

\section{REFERENCES}

[1] Nabina N Rawther, JiniCheriyan, "Detection and Classification of Cardiac Arrhythmias based on ECG and PCG using Temporal and Wavelet features," IJARCCE., vol.4, Issue 4, April. 2015.

[2] AtiyeRiasi, Maryam Mohebbi, "Prediction of Ventricular Tachycardia Using Morphological Features Of ECG Signal,” 2015 International Symposium On Artificial Intelligence and Signal Processing(AISP).

[3] Sina Mohammad-Taheri, Mohammad-Ali Masnadi, Amin Rafiezade, "Slope Analysis Based Methods for Detection of Ventricular Fibrillation and Ventricular Tachycardia," 2016 $24^{\text {th }}$ Iranian Conference On Electrical Engineering(ICEE).

[4] Eduardo Jose da S.Luz, William Robson Schwartz, Guillermo Camara Chavez, David Menotti, "ECG based Heartbeat Classification for Arrhythmia Detection: A Survey," Computer methods and programs in biomedicine, December 2015.

[5] NuryaniNuryani, BambangHarjito, IwanYahya, Anik Lestari, "Atrial Fibrillation Detection Using Support Vector Machines," Joint ICEVT \& IMECE 2015.

[6] R.Rodriguez, A. Mexicano, J.Bila, S.Cervantes, "Feature extraction of electrocardiogram signals by applying adaptive threshold and principle component analysis," Journal of Applied Research and Technology, 13(2015) 26-269

[7] Durgesh Kumar Ojha, Monica Subashini, "Analysis of Electrocardiograph(ECG) Signal for the Detection of Abnormalities Using MATLAB," International Journal of Medical, Health, Biomedical, Bioengineering and Parmaceutical., vol.8, No:2, 2014.

[8] Physionet Database website. [Online]. Available: http://www.physionet.org/

\section{BIOGRAPHIES}

Palimaru Aparna, received her B.E (E\&E) degree from NMAM Institute of Technology, Nitte, Karkala, India in

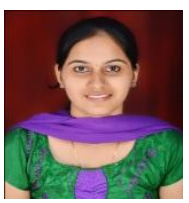
2015. She is pursuing her M.Tech (Microelectronics and Control Systems) in NMAM Institute of Technology, Nitte, Karkala, India, under Visvesvaraya Technological University, Belgavi. Her areas of interestare Control Systems, Embedded Systems.

Praveen Mirajkar,received his B.E (E\&C) degree from Bapuji Institute of Engineering \& Technology, Davangere, India in 2013. He received hisM.Tech (Microelectronics

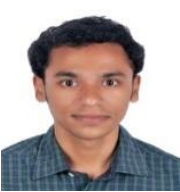
and Control Systems) in PES Institute of Technology, Bangalore, India, under Visvesvaraya Technological University, Belgavi in 2015. He is currently working as R\&D Engineer at Caliber Assurance Services, Bangalore. His areas of interest are Microcontroller, Logic Design, signal and Image processing.

Raghavendra Prabhu, received his M.Tech (Microelectronics and Control Systems) in NMAM Institute of Technology, Nitte, Karkala, India, under

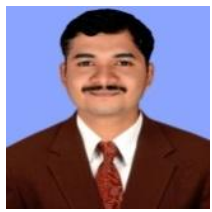
Visvesvaraya Technological University in Belgavi in 2015. He is currently working as Assistant Professor in the department of E\&E, NMAMIT, Nitte. His areas of interest are Embedded System \& Renewable Energy. 